\title{
Melatonin as an Agent for Cardioprotection in Patients with ST-Elevation Myocardial Infarction and Short Ischaemic Time
}

\author{
Alberto Dominguez-Rodriguez ${ }^{1,2}$ - Pedro Abreu-Gonzalez ${ }^{3} \cdot$ Russel J. Reiter $^{4}$
}

Published online: 18 January 2017

(C) Springer Science+Business Media New York 2017

\section{To the Editor}

We were interested in the Review article written by Kloner et al., about of the cardioprotection in patients with acute myocardial infarction. The authors should be congratulated for this excelent review. The authors discuss the recent successes as well as recent disappointments, and describe some of the newer potential therapies in cardioprotection in patients with acute myocardial infarction [1]. However, we would like to point out that there is a recent pharmacological therapy which has been investigated in the clinical setting to target myocardial ischemia reperfusion injury in reperfused ST-segment elevation myocardial infarction (STEMI) patients [2].

The ubiquitous distribution and functional diversity of melatonin as currently envisioned far exceeds that of original expectations [3]. In particular, due to definitive studies within the last 15 years, melatonin has been linked to a wide range of functions including anti-inflammation, antioxidant, oncostatic, circadian rhythm regulation, etc. Melatonin has been shown to decrease nocturnal hypertension, reduce the pulsatility index in the internal carotid artery, decrease platelet

Alberto Dominguez-Rodriguez

adrvdg@hotmail.com

1 Department of Cardiology, Santa Cruz de Tenerife, Hospital Universitario de Canarias, Ofra s/n La Cuesta, E-38320 Tenerife, Spain

2 Facultad de Ciencias de la Salud, Universidad Europea de Canarias. La Orotava, Santa Cruz de Tenerife, Spain

3 Departamento de Ciencias Médicas Básicas (Unidad de Fisiología), Universidad de La Laguna, Santa Cruz de Tenerife, Spain

4 Department of Cellular and Structural Biology, University of Texas Health Science Center, San Antonio, TX, USA aggregation, and reduce serum catecholamine levels [4]. Moreover, decreased melatonin levels were reported in various pathological conditions including hypertension with nondipper pattern, congestive heart failure, ischemic heart disease, and in patients after acute myocardial infarction [4, 5]. We point out that there have been several recent systematic reviews on this topic that the reader might find useful [4, 6-8].

Recently, our group published the methodology and results of MARIA (Melatonin Adjunct in the acute myocaRdial Infarction treated with Angioplasty trial), a randomized, double-blind, placebo controlled trial [9]. Briefly, patients were recruited in three Spanish centers. The investigational product was a formulation of melatonin in polyethylene glycol solution. Patients randomized to melatonin received a dose of $51.7 \mu \mathrm{mol}$ given at a time period of $60 \mathrm{~min}$ starting immediately before primary percutaneous coronary intervention (pPCI) and a bolus of $8.6 \mu \mathrm{mol}$ of intracoronary melatonin given through the pPCI-guiding catheter within the first $60 \mathrm{~s}$ after restoring the blood flow to the infarct related artery. The control group received a matching placebo formulation. The primary endpoint was myocardial infarct size measured by magnetic resonance imaging which was performed within 1 week after pPCI [9]. In our study, melatonin had an acceptable safety and tolerability profile. However, melatonin did not appear to exert a significant effect on myocardial infarct size. Moreover, it may have a detrimental effect after STEMI, mainly because it might facilitate left ventricular remodeling [9]. We speculate two possible mechanisms: (1) Very high doses of melatonin into the coronary circulation possibly led to loss of cardioprotection; to date, no study has been undertaken to assess the optimal dose of melatonin for cardioprotection during myocardial reperfusion. A second potential explanation is, (2) the median pain-to- 
balloon time was so long that it likely negated the benefits of melatonin.

There is experimental evidence that the mechanisms responsible for reperfusion-induced cell death differ according to the duration of ischemia. While the inhibition of mitochondrial permeability transition by postconditioning may be effective after a prolonged ischemic period, treatments that reduce mitochondrial dysfunction appear to be more effective after shorter ischemic periods [10]. The effect of melatonin in relation to duration of ischemia was not a part of the original statistical analysis plan, but the hypothesis came to our attention after the parent study was published. A post hoc analysis of the outcome of the MARIA trial demonstrated that melatonin given $<2.5 \mathrm{~h}$ after symptom onset reduced myocardial infarct size by approximately $40 \%$ as measured by magnetic resonance imaging. The results of this study suggest that melatonin administered earlier may result in a greater cardioprotective effect compared with delayed administration and this finding, although hypothesis-generating, has potential clinical implications for the treatment of patients with STEMI (manuscript submitted).

We hope this letter to the editor provides a worthy account of the huge importance and impact the discovery of melatonin has made in the field of cardiovascular research over the last 20 years.

\section{Compliance with Ethical Standards}

Funding This editorial was supported by Institute Carlos III (ISCIII) (PI15 / 01260), General Branch Evaluation and Research Promotion, State Plan of Scientific and Technical Research and Innovation 20132016 and European Regional Development Fund Health - FEDER-, which relates to the topic of this study.
Conflict of Interest The authors report that they have no relationships relevant to the contents of this paper to disclose.

\section{References}

1. Kloner RA, Hale SL, Dai W, Shi J. Cardioprotection: Where to from here? Cardiovasc Drugs Ther. 2017. (in press)

2. Dominguez-Rodriguez A, Abreu-Gonzalez P, Reiter RJ. Melatonin for cardioprotection in ST elevation myocadial infarction: are we reading for the challenge? Heart. 2017. (in press)

3. Reiter RJ, Tan DX, Galano A. Melatonin: exceeding expectations. Physiology (Bethesda). 2014;29:325-33.

4. Pandi-Perumal SR, BaHammam AS, Ojike NI, et al. Melatonin and human cardiovascular disease. J Cardiovasc Pharmacol Ther. 2017. (in press)

5. Dominguez-Rodriguez A, Abreu-Gonzalez P, Avanzas P. The role of melatonin in acute myocardial infarction. Front Biosci (Landmark Ed). 2012;17:2433-41.

6. Reiter RJ, Mayo JC, Tan DX, Sainz RM, Alatorre-Jimenez M, Qin L. Melatonin as an antioxidant: under promises but over delivers. J Pineal Res. 2016;61:253-78.

7. Opie LH, Lecour S. Melatonin has multiorgan effects. Eur Heart J Cardiovasc Pharmacother. 2016;2:258-65.

8. Simko F, Baka T, Paulis L, Reiter RJ. Elevated heart rate and nondipping heart rate as potential targets for melatonin: a review. J Pineal Res. 2016;61:127-37.

9. Dominguez-Rodriguez A, Abreu-Gonzalez P, De la TorreHernandez JM, et al. Effect of intravenous and intracoronary melatonin as an adjunct to primary percutaneous coronary intervention for acute ST-elevation myocardial infarction: results of the Melatonin Adjunct in the acute myocaRdial Infarction treated with Angioplasty (MARIA) trial. J Pineal Res. 2017;62.

10. Hausenloy DJ, Botker HE, Engstrom T, et al. Targeting reperfusion injury in patients with ST-segment elevation myocardial infarction: trials and tribulations. Eur Heart J. 2017. (in press) 